\title{
Collective dynamics and conformal ordering in electrophoretically driven nematic colloids
}

\author{
Arthur V. Straube, ${ }^{1,2,3, *}$ Josep M. Pagès,,${ }^{4, *}$ Pietro Tierno $\odot,{ }^{3,5,6, \dagger}$ Jordi Ignés-Mullol $\odot,{ }^{4,5, \ddagger}$ and Francesc Sagués ${ }^{4,5}$ \\ ${ }^{1}$ Department of Mathematics and Computer Science, Freie Universität Berlin, Berlin, Germany \\ ${ }^{2}$ Group “Dynamics of Complex Materials”, Zuse Institute Berlin, Berlin, Germany \\ ${ }^{3}$ Departament de Física de la Matèria Condensada, Universitat de Barcelona, Barcelona, Spain \\ ${ }^{4}$ Departament de Ciència de Materials i Química Física, Universitat de Barcelona, Spain \\ ${ }^{5}$ Institut de Nanociència i Nanotecnologia, IN ${ }^{2} U B$, Universitat de Barcelona, Barcelona, Spain \\ ${ }^{6}$ Universitat de Barcelona Institute of Complex Systems (UBICS), Universitat de Barcelona, Barcelona, Spain
}

(Received 19 April 2018; revised manuscript received 29 April 2019; published 30 September 2019)

\begin{abstract}
We present a theoretical framework to understand the collective dynamics of an ensemble of electrophoretically driven colloidal particles that are forced to assemble around a single topological defect in a nematic liquid crystal by an alternating current electric field. Our generic model combines phoretic propulsion with electrostatic interactions and liquid-crystal-mediated hydrodynamics, which are effectively cast into a long-range interparticle repulsion, while nematic elasticity plays a subdominant role. Simulations based on this model fully capture the collective organization process observed in the experiments and other striking effects as the emergence of conformal ordering and a nearly frequency-independent repulsive interaction above $10 \mathrm{~Hz}$. Our results demonstrate the importance of hydrodynamic interactions on the assembly of driven microscale matter in anisotropic media.
\end{abstract}

DOI: 10.1103/PhysRevResearch.1.022008

Nematic liquid crystals (NLCs) are viscoelastic fluids that play a key role in modern technologies, being present in electronic displays, switches, and thermal and optical sensors [1]. NLCs typically present rod-shaped molecules with a long-range orientational order around a mean director field $\hat{\boldsymbol{n}}(\boldsymbol{r})$. When dispersed in a NLC, a colloidal particle produces an elastic distortion of $\hat{\boldsymbol{n}}$ with the appearance of a topological point or line defect around its surface [2-5]. In contrast to isotropic fluids, in nematic media elastic interactions mediated by topological defects dominate the organization of microscale matter at equilibrium. These interactions have been used as an efficient strategy to precisely control and guide the colloidal self-assembly [6-10] and thus have been thoroughly investigated in the past [11-15]. The situation, however, could change when considering out-of-equilibrium systems, as the case of particles driven by external fields, where different interactions competing with the elastic ones may affect the system dynamics significantly. Recent advances in the field showed the possibility of manipulating and transporting colloidal particles with point defects in an NLC using an oscillating electric field [16]. The mechanism of motion is based on the induced-charge electroosmosis (ICEO), where the oscillating field induces a periodic ionic flow and electric charge accumulation around the particles. In the simplest case,

\footnotetext{
${ }^{*}$ These authors contributed equally to this work.

†ptierno@ub.edu

†ignes@ub.edu
}

Published by the American Physical Society under the terms of the Creative Commons Attribution 4.0 International license. Further distribution of this work must maintain attribution to the author(s) and the published article's title, journal citation, and DOI. the presence of a single point defect on the particle surface breaks the spatial symmetry of the flow and generates a net translational motion. A further study allowed visualization of the electrokinetic flow [17], suggesting that long-range hydrodynamic interactions may play an important role in such a system. While these interactions have been considered as essential to understand the dynamics in different physical systems, from active matter [18] to microfluidics [19], they are usually relegated to play a secondary role when considering interacting multibody systems in anisotropic media.

In this context, we recently observed the formation of stationary clusters when interacting nematic colloids are phoretically assembled around a topological defect photoinduced above a plate in a thin nematic cell $[20,21]$. The transport technique is based on ICEO and provides a battery of fascinating experimental results with emergent complex structures in the form of aster and spiral assemblies, allowing the transport of both liquid and solid inclusions [22]. These results prompt the current Rapid Communication, aimed at providing a theoretical understanding of these collective phenomena and the observation of conformal ordering. Clustering effects in active colloidal systems have received much attention in isotropic [23-26] and anisotropic [27,28] fluids. However, few studies have focused on assemblies resulting from driven systems in NLC [20,29], and no one reported clusters displaying conformal ordering. Checking our theory against previous experimental data offers important insights. At the same time, we show that the key ingredients, corresponding to propulsion towards a center of attraction and interparticle repulsion, remain relevant to broader contexts.

We briefly describe the experimental system, illustrated in Figs. 1(a)-1(c). We use polystyrene pear-shaped particles (PNT004UM Magsphere Inc.) having width and length of 3 and $4 \mu \mathrm{m}$, respectively [Fig. 1(b)]. The particles are dispersed 

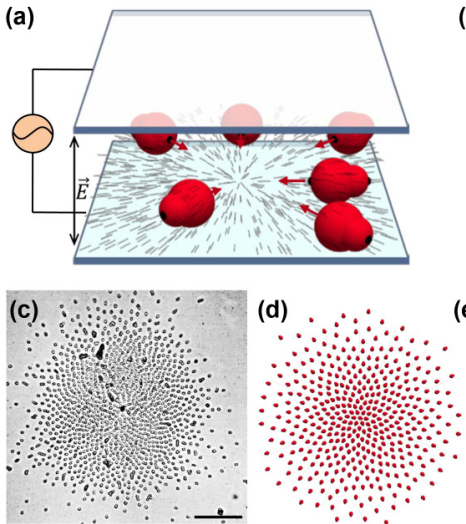

(b)

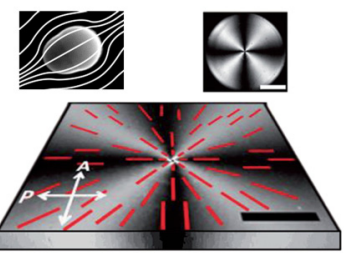

(e)

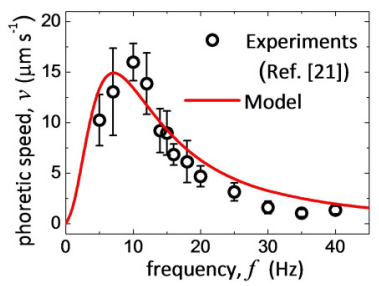

FIG. 1. (a) Schematic of the experimental system, with anisometric colloids driven in the NLC by an oscillating electric field normal to the sample. (b) Polarization microscope image under crossed polarizer (P) and analyzer (A) of the imprinted NLC aster pattern. Dashed red lines indicate the NLC orientation. Scale bar is $200 \mu \mathrm{m}$. Top: electron microscope image of a single particle with the NLC field lines due to planar anchoring, adapted from Ref. [20]. (c) Experimental image of one assembled cluster under $E_{0}=0.76 \mathrm{~V}_{\mu \mathrm{m}^{-1}}$ and $f=20 \mathrm{~Hz}$. Scale bar is $50 \mu \mathrm{m}$, from Ref. [21]. (d) Simulated cluster assembly for parameters as in the experiments. (e) Particle phoretic speed $v_{0} v s$ frequency $f$ for $E_{0}=0.76 \mathrm{~V} \mu \mathrm{m}^{-1}$. Solid line is a fit to the data (see text). Error bars are obtained from the statistical analysis of different experimental realizations.

in a NLC (MLC7029, Merck) characterized by a negative dielectric anisotropy, $\Delta \varepsilon=-3.6$ (at $1 \mathrm{kHz}$ ) and thus the nematic orients perpendicularly to an applied electric field, $\boldsymbol{E}$. The nematic and the particles are sandwiched between two conducting plates (coated with indium tin oxide), one with homeotropic anchoring, while the other is functionalized with a photosensitive self-assembled azosilane (AZ) monolayer [30]. Once in the NLC, the particles force a planar (parallel) anchoring of the NLC, thus orienting their long axis along the local $\hat{\boldsymbol{n}}(\boldsymbol{r})$. As shown in Fig. 1(a), when $\boldsymbol{E}$ is applied along the $\hat{z}$ direction, $\hat{\boldsymbol{n}}(\boldsymbol{r})$ reorients toward the $(\hat{\boldsymbol{x}}, \hat{\boldsymbol{y}})$ plane, forcing the particles to align their long axis in that plane. As shown in Fig. 1(b), ultraviolet (UV) light is used to force the NLC in contact with the AZ layer to adopt an aster-like planar configuration that converges to a central point defect of charge $+1 .{ }^{1}$

Application of the electric field between the two plates, $\boldsymbol{E}=E_{0} \cos (2 \pi f t) \hat{z}$, induces in-plane reorientation of the NLC and electro-osmotic flows of ions around the particles. The anisometric shape of the particles breaks the spatial symmetry, unbalancing the ionic flows and leading to net phoretic propulsion at a speed $v_{0}$. Dispersed particles follow the director field locally and ballistically converge toward the point defect. During motion, individual particles display negligible thermal fluctuations (self-diffusion coefficient in the absence of field $D \sim 10^{-3} \mu \mathrm{m}^{2} \mathrm{~s}^{-1}$ ) and their average speed $v_{0}$ depends explicitly on the driving frequency $f$

\footnotetext{
${ }^{1}$ Because of topological constraints, the $s=+1$ defect is balanced by a negative $s=-1$ defect that arises outside the observation view of the experiment.
}

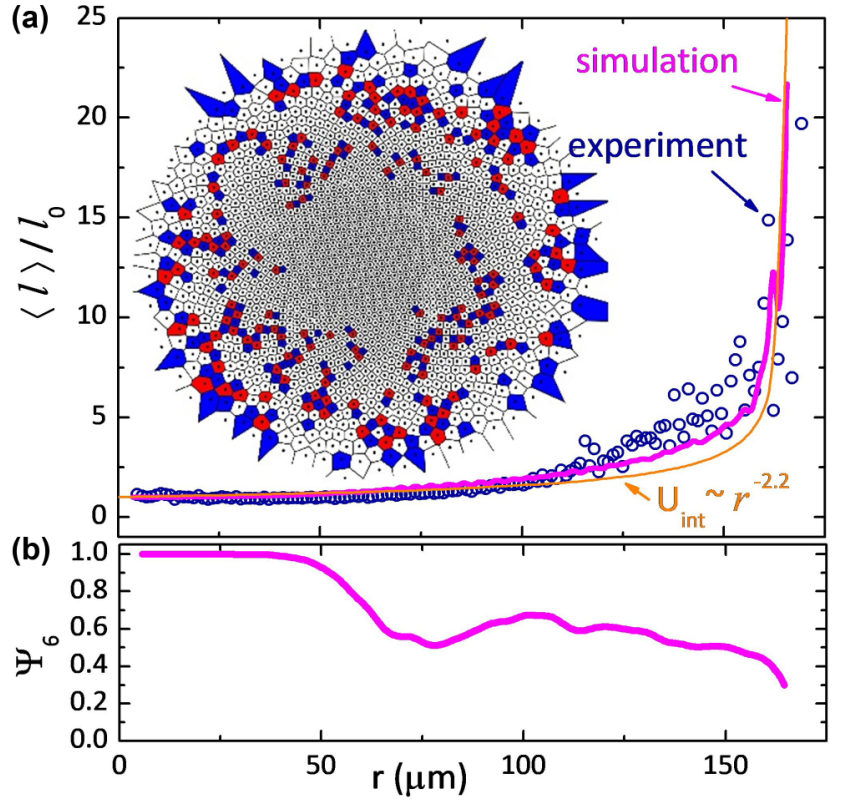

FIG. 2. (a) Average lattice spacing $\langle l\rangle$ from a Voronoi tessellation $v s$ radial position $r$ in the conformal crystal. Here, $l_{0}$ denotes the minimum lattice distance in the cluster, $l_{0}=4.1 \mu \mathrm{m}\left(l_{0}=4.3 \mu \mathrm{m}\right)$ for simulation (experiment). Scattered points are experimental data (from Ref. [21]), the thick magenta line is a simulation, and the thin orange line is a fit following Eq. (1). Inset: Voronoi triangulation of a simulated aster. Plaquettes with sixfold coordination are left white, while those with seven-(five-)fold coordinations are filled in red (blue). (b) Magnitude of orientational order parameter $\Psi_{6}(r)=$ $\frac{1}{N_{k}}\left|\sum_{j=1}^{N_{k}} \mathrm{e}^{6 \mathrm{i} \theta_{k j}}\right|$ computed from simulation; $\theta_{k j}$ is the bond angle between particles $k$ and $j$, and $N_{k}$ is the number of nearest bonds formed by a particle at position $r$.

[Fig. 1(e)]. Following our derivation [31], $v_{0}(f)=u_{0} \alpha(f)$ with the maximum speed $u_{0}$ and the dimensionless frequencydependent function, $\alpha(f)=\frac{\left(\tau_{\mathrm{s}}+\tau_{\mathrm{e}}\right)^{2} \omega^{2}}{\left(1+\tau_{\mathrm{s}}^{2} \omega^{2}\right)\left(1+\tau_{\mathrm{e}}^{2} \omega^{2}\right)}$. Here, $\omega=2 \pi f$, $\alpha(\omega) \in[0,1], \eta$ is an average NLC viscosity, and $\tau_{\mathrm{s}}$ and $\tau_{\mathrm{e}}$ are the particle and the electrode charging times, respectively. As shown in Fig. 1(e), by fitting the propulsion speed of individual particles, we determine both timescales simultaneously and obtain $u_{0}=15 \mu \mathrm{m} \mathrm{s}^{-1}, \tau_{\mathrm{s}}=0.016 \mathrm{~s}$, and $\tau_{\mathrm{e}}=0.032 \mathrm{~s}$, similar to those found in separate experiments [22].

Considering collective effects, experiments and simulations show that in the steady state, the colloidal particles assemble around the topological defect forming compact clusters [Figs. 1(c) and 1(d)], with the maximum size set by the colloidal fraction within the sample. From particle tracking, we find that the average lattice spacing $\langle l\rangle$, determined from a Voronoi tessellation of the particle positions, increases nonlinearly from the center to the periphery. Strikingly, the arrested structure displays a conformal ordering [32] as shown in Fig. 2(a). Conformal lattice can be obtained from a regular crystal in the complex plane via a conformal transformation. Conformal geometries have been observed in different two-dimensional systems in condensed matter, when adding a long-range repulsion to a confining potential. Examples include dusty-plasma [33,34], quantum dots [35], electrons on liquid helium [36], or pinning in high- $T_{c}$ superconductors [37]. Similar to these cases, the emergence of 
conformal ordering in our system suggests the existence of a strong pairwise repulsion that prevents aggregation between the particles. To gain further insight into this interaction, we assume its algebraic (long-range) nature and estimate its spatial dependence. For an interaction potential of the form $U_{\text {int }}=r^{-m}(m>2)$ and an external confining potential $U_{\text {ext }}=$ $\kappa|r|^{n}(\kappa>0$ and $n \geqslant 1)$, the average lattice spacing can be derived as [38]

$$
\frac{\langle l\rangle}{l_{0}}=\left[1-\left(\frac{r}{R}\right)^{n}\right]^{-1 / m}
$$

with $R=R\left(m, n, l_{0}, \kappa\right)$ is the total radius of the cluster and $l_{0}$ being the lattice distance at its center. We use Eq. (1) to fit the experimental data, keeping $n=1$, which corresponds to the constant phoretic propulsion force, and varying $m$ and $R$. From the fit, we obtain $R=166 \mu \mathrm{m}$, similar to the experimental one in Ref. [21], and an exponent $m=2.2 .^{2}$ We note that other values of $m, n$ give unphysical results for $R$. This analysis is generally consistent with the existence of a homogeneous phoretic propulsion and a long-range repulsion. Our clusters also present defect patterns and "scars," namely sequences of alternating five- and seven-fold disclinations. These scars are observed to circulate the cluster periphery [inset in Fig. 2(a)] rather than terminating in the center as observed in repulsive electrostatic colloids [38]. To quantify the ordering, we measure the amplitude of the bond order parameter $\Psi_{6}$ from the particle positions. As shown in Fig. 2(b), the emergence of orientational disorder $\left(\Psi_{6}<1\right)$ corresponds to the locations of the scars and increases toward the cluster edge, where the particles have fewer neighbors.

To understand this colloidal assembly, we perform numerical simulations considering a monolayer of $N$ particles approximated as hard spheres confined to a plane and driven along the local orientation of a nematic director field. In analogy with the experiments, we consider that the director features a radial aster pattern emanating from a single topological point defect, $\hat{\boldsymbol{n}}(\boldsymbol{r})=-\hat{\boldsymbol{r}}$ [39]. We assume that the particles are propelled at a constant, frequency-dependent speed $v_{0}(f)$ arising from the ICEO flow, while the interactions with neighboring particles modulate the instantaneous speed. Similarly to Ref. [40] (see also Ref. [31]), we include the hydrodynamic flows into an effective pairwise interaction, and we assume that the motion of a particle $i=1 \ldots N$ at position $\boldsymbol{r}_{i}=\left(x_{i}, y_{i}\right)$ is governed by the overdamped equation

$$
\frac{d \boldsymbol{r}_{i}}{d t}=v_{0, i}(f) \hat{\boldsymbol{n}}\left(\boldsymbol{r}_{i}\right)-\frac{1}{\gamma} \sum_{j \neq i} \frac{\partial U}{\partial \boldsymbol{r}_{i}},
$$

where $\gamma$ is the friction coefficient and $U$ is the total pairwise interaction potential, all terms of which are explained below. For a derivation, see the Supplemental Material [31].

The first term $U_{\mathrm{hd}}$ is a long-range hydrodynamic repulsion that results from the electroosmotic flows generated around each particle. For phoretic particles with planar anchoring, recent experiments [17] revealed the quadrupolar structure of

\footnotetext{
${ }^{2}$ The fact that we have $m=2.2$ and not exactly 2 as required by the hydrodynamically induced repulsive potential is attributed to the presence of another (electrostatic) repulsion mechanism with $m=3$.
}
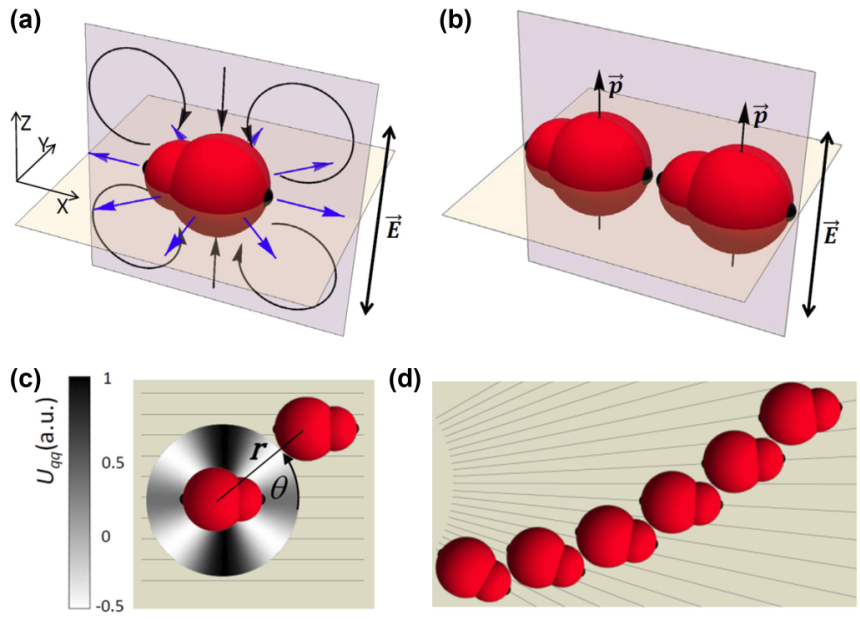

FIG. 3. (a) Hydrodynamic repulsion due to phoretic flows, which are pushed away from the particles in the plane of the assembly (blue arrows). Local director field is along the $\hat{x}$ axis. (b) Induced electric dipoles are parallel, coplanar, and perpendicular to the plane of the assembly, resulting in a net repulsion. (c) Anisotropic NLC-mediated elastic quadrupolar interactions, $U_{\mathrm{qq}}$, become attractive when the particles' centers are shifted by $\approx 45^{\circ}$ relative to the local NLC director (lines), promoting the formation of arches (d).

such flows. These findings, together with the fact that our NLC has negative dielectric anisotropy and the electric field is perpendicular to the plane of particle motion, suggest that the induced flows move radially outward from each particle in the plane of the assembly, independently of the in-plane direction, Fig. 3(a). Although our particles are not perfectly spherical, the assumption of in-plane isotropic flows will be valid in the far field [31]. Since these osmotic flows are the same as those that provide the phoretic propulsion, their dependence on the driving frequency is the same as that determined for the particle speed [Fig. 1(e)]. Far-field hydrodynamic velocities in cell geometries (with either an isotropic solvent or a NLC) have been shown to decay as $r^{-3}[17,41]$. As two particles approach, the overlap of their surrounding radial flows results in an in-plane net repulsive force that gives rise to the repulsive potential

$$
U_{\mathrm{hd}}(r, \omega)=\frac{A}{r^{2}} \frac{\left(\tau_{\mathrm{s}}+\tau_{\mathrm{e}}\right)^{2} \omega^{2}}{\left(1+\tau_{\mathrm{s}}^{2} \omega^{2}\right)\left(1+\tau_{\mathrm{e}}^{2} \omega^{2}\right)},
$$

with $A$ being a constant prefactor.

The second contribution, whose need will be justified later, is an electrostactic term $U_{\mathrm{dd}}$ that arises from the induced dipole moments $\boldsymbol{p}$ within the particles. Application of the electric field induces periodic charge separation and formation of identical switching dipoles in all particles. The resulting repulsive pairwise interaction can be expressed as $U_{\mathrm{dd}}(r, \omega) \propto$ $\boldsymbol{p}^{2} / \boldsymbol{r}^{3}[31,42,43]$,

$$
U_{\mathrm{dd}}(r, \omega)=\frac{B}{r^{3}} \frac{\left(1 / 4+\tau_{\mathrm{s}}^{2} \omega^{2}\right) \tau_{\mathrm{e}}^{2} \omega^{2}}{\left(1+\tau_{\mathrm{s}}^{2} \omega^{2}\right)\left(1+\tau_{\mathrm{e}}^{2} \omega^{2}\right)},
$$

with $B$ being a frequency-independent prefactor.

The near field does not seem to affect the assembly, and we include hard-core repulsion, $U_{\mathrm{hc}}$, with a hydrodynamic diameter of the particles slightly larger than their real size. 

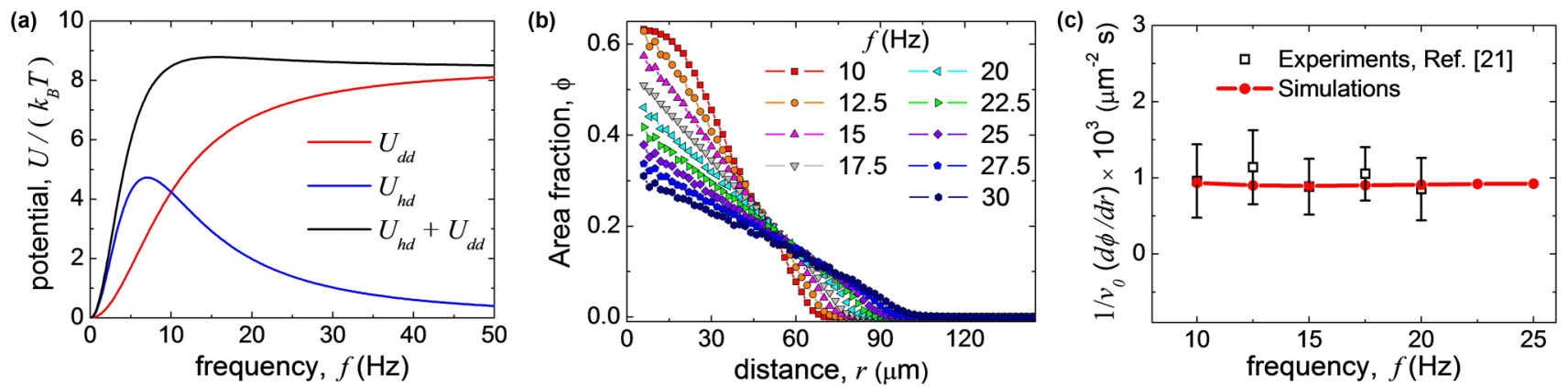

FIG. 4. (a) Hydrodynamically induced $\left(U_{\mathrm{hd}}\right.$, blue), dipolar $\left(U_{\mathrm{dd}}\right.$, red $)$, and combined $\left(U_{\mathrm{hd}}+U_{\mathrm{dd}}\right.$, black) repulsion potentials, calculated for a center-to-center distance of $10 \mu \mathrm{m}$ and normalized by the thermal energy $k_{\mathrm{B}} T(T=293 \mathrm{~K})$. (b) Simulated area fraction $\phi$ occupied by the particles $v s$ distance from the center of the cluster for different frequencies. (c) Comparison between experiments and simulations: slope of the linear region of the density profiles $\phi(r)$ normalized by the phoretic speed $v_{0} v s$ driving frequency $f$ extracted from the experiments (open symbols) and simulations (filled symbols). Error bars are obtained from the statistical analysis of different experimental realizations.

Indeed, in the experiment the particles in a cluster do not come into contact, which can be due to local defects or thin boundary layerlike vortex flows attached to the surface of the particles $[17,44]$.

For completeness, we also include the effective elasticity of the NLC, which is responsible for the optimal particle arrangement in the denser parts of the cluster, by means of the quadrupolar interaction, $U_{\mathrm{qq}}(\boldsymbol{r}, \hat{\boldsymbol{n}})=\mathrm{Cr}^{-5}(3-$ $\left.30 \cos ^{2} \vartheta+35 \cos ^{4} \vartheta\right)$, where $\vartheta=\vartheta(\boldsymbol{r}, \hat{\boldsymbol{n}})$ is the angle between the vector connecting the centers of particles and the far-field orientation of the nematic director, Fig. 3(c). This interaction is frequency independent and has a shorter spatial range than the other long-range repulsive interactions. The dominance of quadrupolar elasticity, promoted by the planar NLC anchoring on the particle's surface, is evidenced by the off-centered chaining. Dominance of dipolar elastic interactions, which may be favored by the nonspherical particle shape, would result in chaining parallel to the local director, which is not observed $[5,15]$. Combined with the underlying radial NLC director, elasticity results in the formation of arched chains, as shown in Fig. 3(d).

To validate our theory, we compare its predictions with previous experimental results [20,21]. Because of balance between the phoretic propulsion and an effective interparticle repulsion, the particle area fraction $\phi(r)$ was seen experimentally to exhibit a linear decrease [21], reaching a zero value outside the cluster area. Counterintuitively, although the individual propulsion displays a clear frequency dependence [Fig. 1(e)], the net repulsion was observed to be nearly frequency independent above $\approx 10 \mathrm{~Hz}$ [21], entailing the scaling $\frac{d \phi}{d r}(f) \propto v_{0}(f)$. A one-dimensional cluster model based on Eq. (2) explicitly suggests that $\frac{d \phi}{d r}(f) \propto v_{0}(f) / \frac{d^{2} U}{d r^{2}}$ [31]. Thus, while any of the long-range repulsive potentials alone, $U_{\mathrm{hd}}(r, f)$ or $U_{\mathrm{dd}}(r, f)$, could balance the phoretic drive, they would fail to reproduce the observed frequency scaling of $\frac{d \phi}{d r}(f)$. Interestingly, combining these potentials, which display complementary frequency dependencies, makes their joint contribution $U_{\mathrm{hd}}+U_{\mathrm{dd}}$ not only effectively frequency independent above $10 \mathrm{~Hz}$ as shown in Fig. 4(a) but also consistent with the "intermediate" exponent for the spatial decay of interactions, $2<m=2.2<3$, predicted by the analysis of the conformal lattice (Fig. 2).
Finally, we perform numerical simulations of clusters formed by the assembly of $N=400$ driven spherical particles at different frequencies. The two intrinsic timescales, $\tau_{\mathrm{s}}$ and $\tau_{\mathrm{e}}$, and propulsion strength $u_{0}$ were fixed from the fit in Fig. 1(e). Because of an up to $20 \%$ dispersion in the speed of particles found in experimental ensembles [20], we have included a similar amount of Gaussian noise in the phoretic speed of individual particles. With these parameters, we establish the amplitudes of $U_{\mathrm{hd}}$ and $U_{\mathrm{dd}}$ to ensure the observed frequency independence of the total potential and to quantitatively reproduce the experimental slopes in the density profiles, yielding $A / \gamma=29 \pm 3 \mu \mathrm{m}^{4} / \mathrm{s}$ and $B / \gamma=517 \pm 5 \mu \mathrm{m}^{5} / \mathrm{s}$. To achieve the experimentally observed arched chaining, we set $C / \gamma=150 \mu \mathrm{m}^{7} / \mathrm{s}$. From each simulation, we measure $\phi(r)$ at different frequencies [see Fig. 4(b)], reproducing the experimental density profiles [21]. Moreover, the slope $\frac{d \phi}{d r}$, which is found to be independent of $N$, decreases nonlinearly with $f$, following the experimentally detected scaling law above $f \sim 10 \mathrm{~Hz}^{3}$ By normalizing this slope with $v_{0}(f)$, we find that it indeed becomes frequency independent, in quantitatively good agreement with the experimental data; see Fig. 4(c).

To conclude, we have investigated the nonequilibrium assembly of driven particles around a topological defect in a nematic liquid crystal. Our theoretical approach not only explains and reproduces quantitatively well the experimentally observed conformal ordering, but also unambiguously demonstrates that, for a driven system, hydrodynamic interactions can significantly affect the properties of the selfassembled structures. The latter is in striking contrast to the equilibrium nondriven situations. Furthermore, a posteriori, the model implies the existence of two cooperative mechanisms of long-range repulsion that are different in nature. Remarkably, since the system-specific elastic interactions are eventually nonessential, the model remains fairly general with the propulsion and repulsive interactions as only ingredients, extending its relevance far beyond nematic colloids [45]. While there is a wealth of research in static and driven colloids

\footnotetext{
${ }^{3}$ We note, however, that the account for arched chaining is not necessary for reproducing the frequency scaling.
} 
dispersed in isotropic fluids, and most of the underlying physics is well known, our understanding of colloidal transport and assembly in anisotropic fluids is at its infancy. The work reported here should allow development of rigorous physical models for these fascinating out-of-equilibrium systems.

We thank L. Schimansky-Geier and I. Sokolov for fruitful discussions. A.V.S. was partially supported by the Deutsche Forschungsgemeinschaft (DFG, German Research
Foundation) through the grant SFB 1114, project C01 and from MATH+: the Berlin Mathematics Research Center (under Germany's Excellence Strategy, EXC-2046/1 project ID: 390685689), project EF4-4. J.M.P. acknowledges funding from the European Union's Horizon 2020 Fetopen "AbioMatter", Grant No. 665440. Experiments were funded by MINECO (AEI/FEDER, EU), projects FIS 201341144P and FIS2016-78507-C2-1-P. P.T. acknowledges support from the European Research Council grant agreement No. 811234 .
[1] P. Oswald and P. Pieranski, Nematic and Cholesteric Liquid Crystals: Concepts and Physical Properties Illustrated by Experiments, Liquid Crystals Book Series (Taylor \& Francis, Boca Raton, FL, 2005).

[2] R. W. Ruhwandl and E. M. Terentjev, Phys. Rev. E 55, 2958 (1997).

[3] P. Poulin, H. Stark, T. C. Lubensky, and D. Weitz, Science 275, 1770 (1997).

[4] H. Stark, Phys. Rep. 351, 387 (2001).

[5] I. Muševič, Liquid Crystal Colloids, Soft and Biological Matter (Springer, Cham, Switzerland, 2017).

[6] J.-C. Loudet, P. Barois, and P. Poulin, Nature (London) 407, 611 (2000).

[7] V. G. Nazarenko, A. B. Nych, and B. I. Lev, Phys. Rev. Lett. 87, 075504 (2001).

[8] C. P. Lapointe, T. G. Mason, and I. I. Smalyukh, Science 326, 1083 (2009).

[9] I. Muševič, M. Škarabot, U. Tkalec, M. Ravnik, and S. Žumer, Science 313, 954 (2006).

[10] T. A. Wood, J. S. Lintuvuori, A. B. Schofield, D. Marenduzzo, and W. C. K. Poon, Science 334, 79 (2011).

[11] S. V. Burylov and Y. L. Raikher, Phys. Rev. E 50, 358 (1994).

[12] P. Poulin and D. A. Weitz, Phys. Rev. E 57, 626 (1998).

[13] Y. Gu and N. L. Abbott, Phys. Rev. Lett. 85, 4719 (2000).

[14] I. I. Smalyukh and O. D. Lavrentovich, Phys. Rev. Lett. 90, 085503 (2003).

[15] I. I. Smalyukh, O. D. Lavrentovich, A. N. Kuzmin, A. V. Kachynski, and P. N. Prasad, Phys. Rev. Lett. 95, 157801 (2005).

[16] O. D. Lavrentovich, I. Lazo, and O. P. Pishnyak, Nature (London) 467, 947 (2010)

[17] I. Lazo, C. Peng, J. Xiang, S. V. Shiyanovskii, and O. D. Lavrentovich, Nat. Commun. 5, 5033 (2014).

[18] M. C. Marchetti, J. F. Joanny, S. Ramaswamy, T. B. Liverpool, J. Prost, M. Rao, and R. A. Simha, Rev. Mod. Phys. 85, 1143 (2013).

[19] T. M. Squires and S. R. Quake, Rev. Mod. Phys. 77, 977 (2005).

[20] S. Hernàndez-Navarro, P. Tierno, J. A. Farrera, J. Ignes-Mullol, and F. Sagués, Angew. Chem. Int. Ed. Engl. 53, 10696 (2014).

[21] J. M. Pagès, A. V. Straube, P. Tierno, J. Ignés-Mullol, and F. Sagués, Soft Matter 15, 312 (2019).

[22] S. Hernàndez-Navarro, P. Tierno, J. Ignés-Mullol, and F. Sagués, Soft Matter 9, 7999 (2013).
[23] I. Buttinoni, J. Bialké, F. Kümmel, H. Löwen, C. Bechinger, and T. Speck, Phys. Rev. Lett. 110, 238301 (2013).

[24] J. Palacci, S. Sacanna, A. P. Steinberg, D. J. Pine, and P. M. Chaikin, Science 339, 936 (2013).

[25] F. Ginot, I. Theurkauff, D. Levis, C. Ybert, L. Bocquet, L. Berthier, and C. Cottin-Bizonne, Phys. Rev. X 5, 011004 (2015).

[26] M. Han, J. Yan, S. Granick, and E. Luijten, Proc. Nat. Acad. Sci. USA 114, 7513 (2017).

[27] C. Peng, T. Turiv, Y. Guo, Q.-H. Wei, and O. D. Lavrentovich, Science 354, 882 (2016).

[28] M. M. Genkin, A. Sokolov, O. D. Lavrentovich, and I. S. Aranson, Phys. Rev. X 7, 011029 (2017).

[29] O. P. Pishnyak, S. V. Shiyanovskii, and O. D. Lavrentovich, Phys. Rev. Lett. 106, 047801 (2011).

[30] P. Oswald and J. Ignes-Mullol, Phys. Rev. E 96, 032704 (2017).

[31] See Supplemental Material at http://link.aps.org/supplemental/ 10.1103/PhysRevResearch.1.022008 for one pdf file with details of the theoretical model used in the text.

[32] F. Rothen and P. Pierański, Phys. Rev. E 53, 2828 (1996).

[33] A. A. Koulakov and B. I. Shklovskii, Phys. Rev. B 57, 2352 (1998).

[34] A. Mughal and M. A. Moore, Phys. Rev. E 76, 011606 (2007).

[35] V. Shikin, S. Nazin, D. Heitmann, and T. Demel, Phys. Rev. B 43, 11903 (1991).

[36] C. C. Grimes and G. Adams, Phys. Rev. Lett. 42, 795 (1979).

[37] D. Ray, C. J. Olson Reichhardt, B. Jankó, and C. Reichhardt, Phys. Rev. Lett. 110, 267001 (2013).

[38] V. Soni, L. R. Gómez, and W. T. M. Irvine, Phys. Rev. X 8, 011039 (2018).

[39] A. V. Straube, J. M. Pagès, A. Ortiz-Ambriz, P. Tierno, J. IgnésMullol, and F. Sagués, New J. Phys. 20, 075006 (2018).

[40] D. L. Piet, A. V. Straube, A. Snezhko, and I. S. Aranson, Phys. Rev. Lett. 110, 198001 (2013).

[41] C. Peng, I. Lazo, S. V. Shiyanovskii, and O. D. Lavrentovich, Phys. Rev. E 90, 051002(R) (2014).

[42] V. A. Murtsovkin, Colloid J. 58, 341 (1996).

[43] T. M. Squires and M. Z. Bazant, J. Fluid Mech. 509, 217 (2004).

[44] C. Conklin, O. M. Tovkach, J. Viñals, M. C. Calderer, D. Golovaty, O. D. Lavrentovich, and N. J. Walkington, Phys. Rev. E 98, 022703 (2018).

[45] V. Liljeström, C. Chen, P. Dommersnes, J. O. Fossum, and A. H. Gröschel, Curr. Opin. Coll. Int. Sci. 40, 25 (2019). 